\title{
TIME DOMAIN ANALYSIS OF VARIABILITY IN CYGNUS X-1: CONSTRAINTS ON THE EMISSION MODELS
}

\author{
Thomas J. Maccarone and Paolo S. Coppi \\ Astronomy Department, Yale University, P. O. Box 208101, New Haven, CT 06520-8101 \\ AND \\ JuRi Poutanen \\ Stockholm Observatory, SE-133 36, Saltsjöbaden, Sweden \\ Received 2000 April 21; accepted 2000 June 2; published 2000 July 6
}

\begin{abstract}
We use time domain analysis techniques to investigate the rapid variability of Cygnus X-1. We show that the cross-correlation functions between hard and soft energy bands reach values very close to unity and peak at a lag of less than $2 \mathrm{~ms}$ for energies separated by a factor of 10 . This confirms that the process that produces Xray photons at different energies is extremely coherent on short timescales and strongly constrains emission models proposed to explain Fourier-frequency-dependent time lags. We present autocorrelation functions at different energies and note that their widths decrease with increasing energy. We show that the extended Compton corona model produces autocorrelation functions whose widths increase with increasing energy and that the model of cylindrical waves moving inward through a transition disk has too large a peak lag in the cross-correlation function. Models of magnetic flaring and of drifting blobs in a hot corona can qualitatively fit the observations.
\end{abstract}

Subject headings: accretion, accretion disks — black hole physics - methods: data analysis stars: individual (Cygnus X-1) - X-rays: stars

\section{INTRODUCTION}

The X-ray/ $\gamma$-ray spectrum of an accreting black hole such as Cygnus X-1 in its hard state can be represented as the sum of a few components: a soft component associated with the emission from a cold accretion disk, a hard tail extending up to a few hundred $\mathrm{keV}$ associated with a hot "corona," and a Compton reflection bump produced when hard X-rays are reflected from cold material in the accretion disk (see, e.g., Zdziarski et al. 1997; Gierliński et al. 1997; Poutanen 1998). Spectral data suggest thermal Comptonization by a medium with a temperature of about $100 \mathrm{keV}$ as the origin of the hard tail. The observed X-ray spectral slopes and the amplitude of Compton reflection can be used to determine the geometry of the system. However, a variety of models fit the spectra (see Poutanen 1998; Beloborodov 1999a; Zdziarski 2000), so the parameters of the accretion flow are not well constrained by spectral data alone. Most of the spectral models, however, are applied to time-averaged spectra, despite the fact that the sources show rapid spectral variability (see, e.g., Nolan et al. 1981; Negoro, Miyamoto, \& Kitamoto 1994; Feng, Li, \& Chen 1999), implying rapid changes in the physical conditions. Examining temporal characteristics should help break the degeneracy among spectral models.

Time domain techniques were used in the early days of Xray astronomy before the statistical samples of data were sufficient to make use of Fourier domain analyses (see, e.g., Weisskopf, Kahn, \& Sutherland 1975; Sutherland, Weisskopf, \& Kahn 1978; Priedhorsky et al. 1979; Nolan et al. 1981). The asymmetry of the cross-correlation function (CCF) of Cygnus $\mathrm{X}-1$ was discovered in $\sim 150$ s observations by Priedhorsky et al. (1979) and Nolan et al. (1981). They showed that the CCF peaks at a lag $\leqslant 10-40 \mathrm{~ms}$. Using data from EXOSAT, Page (1985) confirmed these results and claimed an $\sim 6 \mathrm{~ms}$ shift of the peak of the CCF between the 5-14 and 2-5 keV bands. These are the last papers, to our knowledge, that present the CCFs, despite immense advances in temporal resolution, photon statistics, and duration of observations. Aside from attempts to model individual shots (Lochner, Swank, \& Szymkowiak 1991; Negoro et al. 1994; Focke 1998; Feng et al. 1999), recent analyses have concentrated on Fourier domain techniques.

The CCF asymmetry is related to the Fourier-frequencydependent hard time lags between different spectral bands discovered by Ginga (Miyamoto et al. 1988). These data gave new strong constraints on spectral models. However, in the Fourier domain it is difficult to measure time lags at frequencies above $\sim 30 \mathrm{~Hz}$ (see, e.g., Nowak et al. 1999a) that correspond to the light-travel time in a region of the main energy dissipation. On the other hand, in the time domain, the CCFs can be measured accurately down to lags as short as $2 \mathrm{~ms}$. Furthermore, time domain functions (containing, in principle, the same information as their Fourier domain companions) highlight different information that can be used to further constrain emission models.

We present the results of time domain analyses of Cygnus X-1 as observed by the Rossi X-Ray Timing Explorer (RXTE) and compare our observational results with model predictions.

\section{OBSERVATIONS AND RESULTS}

\subsection{Observation Log}

Cygnus X-1 was observed in its hard (low) state 12 times by RXTE during 1997 December, for a total of about $30 \mathrm{ks}$. Here we present results only from the Proportional Counter Array using the standard screening criteria of Earth elevation greater than $10^{\circ}$, offset from a source less than 0.01 , all five proportional counter units on, and the standard time since the last South Atlantic Anomaly passage. For the lowest energies (below $8 \mathrm{keV}$ ), we have data recorded in single-bit (SB) modes, where RXTE counts photons with no spectral information other than whether they fall within the given range of channels. For the higher photon energies, we use the full event mode data with all the available spectral information. The use of SB modes and the subsequent loss of some spectral information were required because of the telemetry limitations of RXTE. We make no background subtractions for our data because the back- 


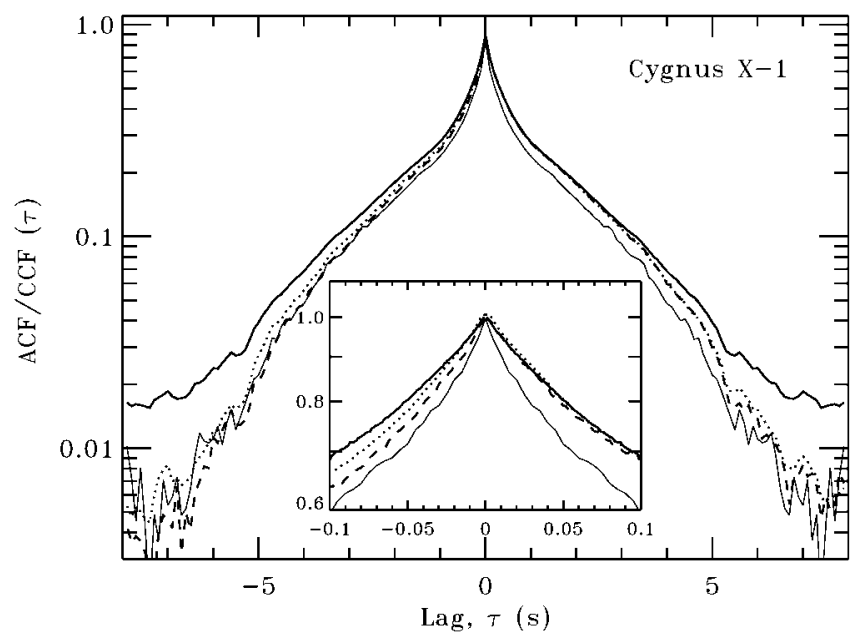

FIg. 1.-Auto- and cross-correlation functions of Cyg X-1 observed in 1997 December. Solid curves show the ACFs for the 2-5 keV energy band (thick curves) and the 24-40 keV band (thin curves). Dotted curves show the CCF between the $8-13 \mathrm{keV}$ band and the $2-5 \mathrm{keV}$ band, and dashed curves represent the CCF for the $24-40 \mathrm{keV}$ band vs. the $2-5 \mathrm{keV}$ band. The CCFs at other energies have similar shapes. The peaks all align at around zero lag. The higher energy curves are narrower. The CCFs are defined in such a way that the peak is expected to appear at a positive lag when hard photons are lagging the soft ones. The errors at small lags are roughly the same size as the line widths and are hence left unplotted for clarity. The CCF peak lag is at less than $2 \mathrm{~ms}$ for the $2-5 \mathrm{keV}$ vs. 8-13 $\mathrm{keV} \mathrm{CCF}$.

ground counts are a very small fraction of the total counts and because current background models for RXTE do not give estimates on timescales shorter than $16 \mathrm{~s}$. RXTE dead time for Cygnus $\mathrm{X}-1$ is about $1 \%$ and should affect only zero-lag bins in the correlation functions.

\subsection{Light Curves}

Using the standard FTOOLS 4.1 software, we extract light curves with $2^{-9} \mathrm{~s}$ resolution $(\sim 2 \mathrm{~ms})$ for each of the SB modes (for energy channels $2-5,5-6$, and 6-8 keV), plus the event mode data binned into three additional energy bands (channels 24-40, 41-71, and 72-132 keV or 8-13, 13-24, and 24-40 keV, respectively). We do not analyze any higher energy data because the background count rates become large above $40 \mathrm{keV}$. We attempted to analyze the data with an $\sim 1 \mathrm{~ms}$ bin size. For the low-energy (and hence higher count rate) bands, the $1 \mathrm{~ms}$ computations allowed us to produce slightly stronger constraints (discussed below). We plot only the $2 \mathrm{~ms}$ binning results in order to make the plots clearer, since the results are essentially the same in either case.

\subsection{Cross-Correlation Functions}

We compute the CCF of Cygnus X-1, comparing each energy band with the lowest energy band. The CCFs are asymmetric, with peaks at lags less than $2 \mathrm{~ms}$ in all cases (see the inset of Fig. 1), and less than $1 \mathrm{~ms}$ between the $3 \mathrm{keV}$ band and bands below $13 \mathrm{keV}$. The rising part of the CCF (soft lags) becomes narrower with energy substantially faster than the decaying part (hard lags). These results agree qualitatively with past results for the CCF (Priedhorsky et al. 1979; Nolan et al. 1981). The CCFs reach values very close to unity, showing that the signal at all the energies is extremely well synchronized. The asymmetry of the CCFs in the time domain has a direct relation to the Fourier-frequency-dependent time lags.
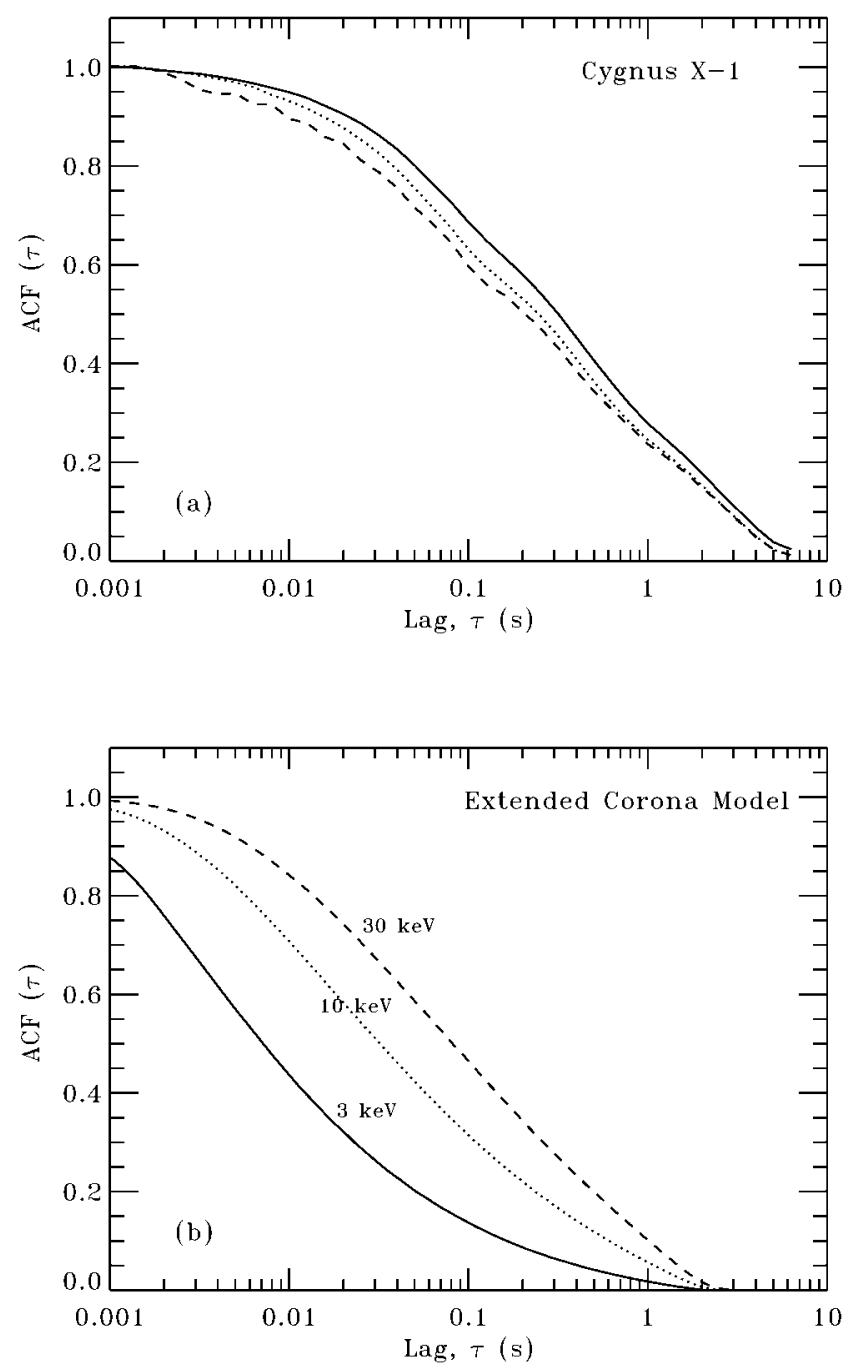

FIG. 2.-ACFs in the different energy bands. (a) Data for Cyg X-1. The solid curves shows the ACF for the $2-5 \mathrm{keV}$ energy band; the dotted curve is for $8-13 \mathrm{keV}$ band, and the dashed curve is for the $24-40 \mathrm{keV}$ band. (b) Prediction of the extended corona model.

\subsection{Autocorrelation Functions}

The autocorrelation functions (ACFs) of Cygnus X-1 are shown in Figures 1 and $2 a$. The width of the ACF decreases with photon energy approximately proportional to $E^{-0.21 \pm 0.01}$ at lags smaller than $\sim 0.3$. (At larger lags the ACFs at different energies are not self-similar.) This strongly constrains the origin of the spectral variability since it requires that the pulses producing the variability last longer at low energies than at higher energies. Similar energy dependence is observed also in the ACF of the peak aligned average shot profiles (Feng et al. 1999). Our results extend their work by demonstrating that the trend of the width of the ACF versus energy applies across more energy bands. More importantly, we prove that this trend is always present in the data and is not subject to the selection effects of a shot-fitting algorithm.

\section{CONSTRAINTS ON THE EMISSION MODELS}

\subsection{Implications on Shot Shapes for Shot Noise Models}

One can attempt to explain the observed ACFs and CCFs in terms of a simple shot noise model (Terrell 1972). Since the 
CCFs peak at a lag $\lesssim 2 \mathrm{~ms}$, the shots at different energies also should reach maxima within $2 \mathrm{~ms}$ from each other. We can develop some intuition about the typical shot profiles by looking at the analytic form of the CCF for shots that have exponential rise and decay and peak at the same time for different energies. The CCF is proportional to

$$
\frac{\tau_{\mathrm{sd}}^{2}\left(\tau_{\mathrm{hr}}+\tau_{\mathrm{hd}}\right) e^{\tau / \tau_{\mathrm{sd}}}}{\left(\tau_{\mathrm{hd}}+\tau_{\mathrm{sd}}\right)\left(\tau_{\mathrm{sd}}-\tau_{\mathrm{hr}}\right)}-\frac{\tau_{\mathrm{hr}}^{2}\left(\tau_{\mathrm{sr}}+\tau_{\mathrm{sd}}\right) e^{\tau / \tau_{\mathrm{hr}}}}{\left(\tau_{\mathrm{hr}}+\tau_{\mathrm{sr}}\right)\left(\tau_{\mathrm{sd}}-\tau_{\mathrm{hr}}\right)}
$$

for negative $\tau$ and proportional to

$$
\frac{\tau_{\mathrm{hd}}^{2}\left(\tau_{\mathrm{sr}}+\tau_{\mathrm{sd}}\right) e^{-\tau / \tau_{\mathrm{hd}}}}{\left(\tau_{\mathrm{sd}}+\tau_{\mathrm{hd}}\right)\left(\tau_{\mathrm{hd}}-\tau_{\mathrm{sr}}\right)}-\frac{\tau_{\mathrm{sr}}^{2}\left(\tau_{\mathrm{hr}}+\tau_{\mathrm{hd}}\right) e^{-\tau / \tau_{\mathrm{sr}}}}{\left(\tau_{\mathrm{sr}}+\tau_{\mathrm{hr}}\right)\left(\tau_{\mathrm{hd}}-\tau_{\mathrm{sr}}\right)}
$$

for positive $\tau$, where $\tau_{\mathrm{sr}}, \tau_{\mathrm{sd}}$ and $\tau_{\mathrm{hr}}, \tau_{\mathrm{hd}}$ are the rise and decay time constants of the shots in soft and hard energy bands, respectively. The proportionality coefficient is $2 /\left[\left(\tau_{\mathrm{sr}}+\tau_{\mathrm{sd}}\right) \times\right.$ $\left.\left(\tau_{\mathrm{hr}}+\tau_{\mathrm{hd}}\right)\right]^{1 / 2}$. The energy dependence of the ACF requires the shots to be shorter at higher energies, i.e., $\tau_{\mathrm{hd}}+\tau_{\mathrm{hr}}<\tau_{\mathrm{sr}}+\tau_{\mathrm{sd}}$. Since the observed CCF (see Fig. 1) is roughly equal to the ACF of the softer energy band for positive $\tau$ and the ACF of the harder energy band for negative $\tau$, and the $\mathrm{CCF}$ rises faster than it decays, one gets $\max \left(\tau_{\mathrm{sd}}, \tau_{\mathrm{hr}}\right) \approx$ $\max \left(\tau_{\mathrm{hr}}, \tau_{\mathrm{hd}}\right)<\max \left(\tau_{\mathrm{sr}}, \tau_{\mathrm{hd}}\right) \approx \max \left(\tau_{\mathrm{sr}}, \tau_{\mathrm{sd}}\right)$. This means that the soft rise timescale is the longest, $\tau_{\mathrm{sr}}>\tau_{\mathrm{sd}}, \tau_{\mathrm{hr}}, \tau_{\mathrm{hd}}$, and that the decay times at different energies are very close to one another (i.e., $\tau_{\mathrm{sd}} \approx \tau_{\text {hd }}$ ) or are so small that the only relevant parameters are the rise times $\left(\tau_{\mathrm{hr}} \gg \tau_{\mathrm{sd}}, \tau_{\mathrm{hd}}\right)$. The energy dependence of the rise time then produces the time lags and the asymmetry of the CCF (see also Miyamoto \& Kitamoto 1989 who arrived at similar conclusions).

In modified shot noise models (see, e.g., Lochner et al. 1991), there is a broad distribution of shot timescales. The shots at different energies should be perfectly synchronized in order to achieve high values of the CCFs. However, constraints on the shape of the shots are not so strong as for the simplest shot noise model. The shots at different energies can have similar properties as described above, or they can be shifted relative to each other (e.g., Poutanen \& Fabian 1999a, 1999b). If the shift depends on the shot timescale being proportional to $\tau^{\alpha}$, one obtains Fourier time lags $\delta t(f) \propto f^{-\alpha}$. In such a case, the $\mathrm{CCF}$ peaks at a lag equal to the delay corresponding to the shortest timescale. The data then constrain the minimum shot timescale to be $\lesssim 1 \mathrm{~ms}$.

\subsection{Extended Corona Models}

Comptonization in a uniform electron cloud produces time lags that are frequency independent in the range we can probe with current X-ray instruments (Miyamoto et al. 1988). This inspired Kazanas, Hua, \& Titarchuk (1997) to propose an extended corona model with an $r^{-1}$ radial density distribution and a size of a few light seconds. The long lags observed at low Fourier frequencies are produced here by photons traveling and scattering over large radii, while the shorter time lags are produced in the central small core of the cloud. The model fits much of the data but has the physical problem of having too much energy input at large radii.

We simulated the light curves for this model and computed the autocorrelation functions at different energies. As an example, we consider a set of parameters from Hua, Kazanas, \& Cui (1999): $k T_{r}=0.2 \mathrm{keV}, k T_{e}=100 \mathrm{keV}, p=1, n_{i}=n_{1}=$ $10^{16} \mathrm{~cm}^{-3}, r_{1}=10^{-3} \mathrm{lt}-\mathrm{s}$, and $r_{2}=10^{3} r_{1}$ (for details, see dis- cussion around their Fig. 1). The shots are assumed to be produced by modulations in the soft flux. Because this model produces delays by having larger light-travel times for higher energy photons, it always produces a wider shot and hence a wider ACF at higher energies (see Fig. 2b). We note that the $\mathrm{ACF}$ at $30 \mathrm{keV}$ in this model is a factor of 10 broader than the $\mathrm{ACF}$ at $3 \mathrm{keV}$, while in the observations it is $50 \%$ narrower. The fact that the ACFs become broader with energy is the intrinsic property of the model, and the problem cannot be resolved by changing the parameters of the system. We conclude that models for which the time lags are produced by light-travel delays can be ruled out.

\subsection{Magnetic Flare Models}

Magnetic flares on the surface of the cold accretion disk were recently shown to produce X-ray/ $\gamma$-ray spectra, in agreement with observations of Cyg X-1 (Beloborodov 1999b). The observed time lags can correspond to the timescale of the evolution of magnetic structures (Poutanen \& Fabian 1999a, $1999 b)$. The magnetic field lines twist because of the differential rotation in the accretion disk and elevate to the corona, releasing magnetic energy and heating the corona. The flare timescale is then of the order of the Keplerian timescale at the relevant distance from the central black hole. Changes in the energy dissipation rate and in the geometry of the flare (e.g., distance from the disk) produce soft-to-hard spectral evolution that is the cause of the hard time lags. A broad distribution of the flare timescale assures that the time lags are inversely proportional to the Fourier frequency $\delta t(f) \propto \tau_{f} \sim 1 /(2 \pi f)$, where $\tau_{f}$ is the shot timescale that contributes to the power spectrum at frequency $f$.

The model of Poutanen \& Fabian (1999b) reproduces well the time lags observed in Cyg X-1, while producing a somewhat wider ACF at higher energies because of the assumption that the energy dissipation rate rises faster than it decays. In the opposite situation, when the dissipation rate rises more slowly than it decays, the ACF and CCF energy dependences can be reproduced easily (see, e.g., Miyamoto \& Kitamoto 1989; Poutanen 2000).

\subsection{Drifting Blob Models}

Böttcher \& Liang (1999) proposed a model in which spectral variability is produced by a cool blob drifting inward through an inhomogeneous hot inner disk. This model qualitatively matches the data. However, the parameters presented in that work produce significant quantitative deviations from the observations in both the Fourier and time domains. While changing the parameters of the system could allow the model to fit the data, a larger problem for this model is that it drives the variability through modulations of the soft photon flux. If the energy dissipation rate in the corona does not change with time, then an increase of the soft photon flux would lead to a softer spectrum and spectral pivoting around $\sim 10 \mathrm{keV}$. The amplitude of the variability would then be a strong function of photon energy (larger at larger energies), and the variability above and below the pivoting point would anticorrelate, contrary to what is observed (see, e.g., Nowak et al. 1999a and § 2.3). In order to reproduce the observed correlated variability at different energies without violating the energy balance, one has to assume that the inward drift of cool blobs is perfectly correlated with the increase of the energy dissipation in the hot corona. It remains to be seen whether such a requirement is physically realistic and whether the model can indeed fit the data. One 
cannot avoid this constraint by proposing an ADAF-type solution in which the coronal cooling rate is dominated by cyclo/synchrotron radiation rather than by Compton cooling. Such a model would require a cyclo/synchrotron luminosity (observed in IR/optical light) far in excess of the X-ray luminosity of $\sim 10^{37} \mathrm{ergs} \mathrm{s}^{-1}$. The observed optical luminosity is only about $5 \times 10^{36} \mathrm{ergs} \mathrm{s}^{-1}$ and is dominated by the companion star.

\subsection{Cylindrical Wave Models}

Cylindrical waves propagating through the accretion disk from the region where the soft X-rays are emitted to the region where the hard X-rays are emitted (e.g., Miyamoto et al. 1988; Kato 1989; Nowak et al. 1999b) have also been proposed as a mechanism for producing hard time lags. The dispersion of wave velocities results in the frequency dependence of the time lags. A recent and relatively well-developed version of such a model (a "transition disk" model) designed to explained the spectrum of Cyg X-1 and the Fourier time lags was recently considered by Misra (2000). However, in this model the peak of the emission at $30 \mathrm{keV}$ is delayed by $\sim 0.015 \mathrm{~s}$ from the peak at $3 \mathrm{keV}$. In such a case, the CCF between the 30 and $3 \mathrm{keV}$ photons would peak at that lag, strongly contradicting the data. One can assume that the low-frequency signal propagates slowly, producing larger time lags, while the highfrequency signal propagates faster, producing smaller time lags. (This is basically a modified shot noise model.) The transition disk model then requires a propagation speed of $\gtrsim 2 c$ in order to fit $\lesssim 2 \mathrm{~ms}$ lag in the $\mathrm{CCF}$.

\section{CONCLUSIONS}

We present the results of the cross-correlation and autocorrelation analysis of the light curve of Cygnus X-1 in several energy bands. The width of the ACF was found to become narrower with photon energy proportional to $E^{-0.2}$. The corresponding $\mathrm{CCFs}$ are asymmetric, but all peak at lags less than $2 \mathrm{~ms}$.

We compare these results to model calculations for an extended Compton corona model, a magnetic flare model, a drifting blob model, and a cylindrical wave model. The extended corona model inherently produces longer shots at higher energies (and, therefore, broader autocorrelation function), and it cannot fit the data even qualitatively. We find that a magnetic flare model can fit all the data if one requires that the energy dissipation rate rises slower than it decays. We also find that while the drifting blob model fits the data qualitatively, it requires the inward drift of cool blobs to be perfectly correlated with the increase of the energy dissipation in the hot corona. A transition disk model predicts a large shift of the peak of the cross-correlation function from zero lag, contradicting the data.

We thank Steve Kahn for useful discussion and Andrei Beloborodov for valuable comments. This research has been supported by NASA grants NAGS-6691 and NAGS-7409 (T. J. M. and P. S. C.) and by the Swedish Natural Science Research Council and the Anna-Greta and Holger Crafoord Fund (J. P.).

\section{REFERENCES}

Beloborodov, A. M. 1999a, in ASP Conf. Ser. 161, High Energy Processes in Accreting Black Holes, ed. J. Poutanen \& R. Svensson (San Francisco: ASP), 295

- 1999b, ApJ, 510, L123

Böttcher, M., \& Liang, E. P. 1999, ApJ, 511, L37

Feng, Y. X., Li, T. P., \& Chen, L. 1999, ApJ, 514, 373

Focke, W. 1998, Ph.D. thesis, Univ. Maryland

Gierliński, M., Zdziarski, A. A., Done, C., Johnson, W. N., Ebisawa, K., Ueda, Y., Haardt, F., \& Phlips, B. F. 1997, MNRAS, 288, 958

Hua, X.-M., Kazanas, D., \& Cui, W. 1999, ApJ, 512, 793

Kato, S. 1989, PASJ, 41, 745

Kazanas, D., Hua, X.-M., \& Titarchuk, L. 1997, ApJ, 480, 735

Lochner, J. C., Swank, J. H., \& Szymkowiak, A. E. 1991, ApJ, 376, 295

Misra, R. 2000, ApJ, 529, L95

Miyamoto, S., \& Kitamoto, S. 1989, Nature, 342, 773

Miyamoto, S., Kitamoto, S., Mitsuda, K., \& Dotani, T. 1988, Nature, 336, 450

Negoro, H., Miyamoto, S., \& Kitamoto, S. 1994, ApJ, 423, L127

Nolan, P. L., et al. 1981, ApJ, 246, 494

Nowak, M. A., Vaughan, B. A., Wilms, J., Dove, J. B., \& Begelman, M. C. 1999a, ApJ, 510, 874

Nowak, M. A., Wilms, J., Vaughan, B. A., Dove, J. B., \& Begelman, M. C. 1999b, ApJ, 515, 726
Page, C. G. 1985, Space Sci. Rev., 40, 387

Poutanen, J. 1998, in Theory of Black Hole Accretion Discs, ed. M. A. Abramowicz, G. Björnsson, \& J. E. Pringle (Cambridge: Cambridge Univ. Press), 100

1000, in X-Ray Astronomy 1999: Stellar Endpoints, AGN and the Diffuse X-Ray Background (Newark: Gordon \& Breach), in press (astro$\mathrm{ph} / 9805025)$

Poutanen, J., \& Fabian, A. C. 1999a, in ASP Conf. Ser. 161, High Energy Processes in Accreting Black Holes, ed. J. Poutanen \& R. Svensson (San Francisco: ASP), 135 . 1999b, MNRAS, 306, L31

Priedhorsky, W., Garmire, G. P., Rothschild, R., Boldt, E., Serlemitsos, P., \& Holt, S. 1979, ApJ, 233, 350

Sutherland, P. G., Weisskopf, M. C., \& Kahn, S. M. 1978, ApJ, 219, 1029

Terrell, N. J., Jr. 1972, ApJ, 174, L35

Weisskopf, M. C., Kahn, S. M., \& Sutherland, P. G. 1975, ApJ, 199, L147

Zdziarski, A. A. 2000, in IAU Symp. 195, Highly Energetic Physical Processes and Mechanisms for Emission from Astrophysical Plasmas, ed. P. C. H. Martens, S. Tsuruta, \& M. A. Weber (San Francisco: ASP), 153

Zdziarski, A. A., Johnson, W. N., Poutanen, J., Magdziarz, P., \& Gierliński, M. 1997, in The Transparent Universe, ed. C. Winkler, T. J.-L. Courvoisier, $\&$ Ph. Durouchoux (ESA-SP 382; Noordwijk: ESA), 373 